\title{
Employing Media Messaging Strategies to Respond to COVID-19 Misinformation
}

\author{
Rachel Field, Gul Saeed, Mariana Villada Rivera, Sabrina Campanella and Lauren Tailor \\ Dalla Lana School of Public Health, University of Toronto, Toronto, Canada \\ E-mail: rachel.field@mail.utoronto.ca
}

Received October 28, 2020

Accepted for publication December 23, 2020

Published online September 5, 2021

\begin{abstract}
Introduction: The COVID-19 pandemic has revealed critical gaps in the public's knowledge of infectious diseases. Experts, including the World Health Organization, acknowledge that an "infodemic" of misinformation is spreading at the same time as the pandemic. Furthermore, $13 \%$ of Canadians age 50 and younger reported using social media as their primary source of information about COVID-19. Thus, in January 2020, the Infectious Disease Working Group (IDWG) was formed by a group of students at the Dalla Lana School of Public Health, University of Toronto. The IDWG's Media Messaging Team (MMT) uses Knowledge Translation (KT) strategies to increase access to evidence-based information related to public health and COVID-19. Specifically, MMT uses virtual platforms, including Twitter and Instagram (@infectious_info), to disseminate information to a wide audience.
\end{abstract}

Objectives: The MMT aims to produce content to dispel pervasive and harmful myths about COVID-19, raise public awareness, and advocate for health equity.

Methods: The team creates 2-4 pieces of original content per week on topics such as Ontario Government legislation updates, myth-busting series, and "Wednesday Series" (summaries of novel research findings). The IDWG employs an equity lens to ensure that the content takes into account the experiences and needs of diverse groups, and that graphics are representative of a diverse audience. Health communication strategies are used to promote audience engagement through compelling and bold content design.

Results: The Instagram account has over 4,400 followers, with some posts surpassing 50,000 views. Qualitative feedback from social media followers indicates that this project is addressing an emerging gap in knowledge resulting from unclear messaging from official bodies, the spread of mis/disinformation, and disparities in health literacy levels.

Conclusions: The findings can inform the development and implementation of KT strategies to reach a wide audience and increase the uptake of public health information. 